\title{
Transcriptional regulation of long-term memory in the marine snail
} Aplysia

\author{
Yong-Seok Lee ${ }^{1}$, Craig H Bailey ${ }^{3}$, Eric R Kandel ${ }^{2,3}$ and Bong-Kiun Kaang*1
}

\begin{abstract}
Address: ${ }^{1}$ National Creative Research Initiative Center for Memory, Department of Biological Sciences, College of Natural Sciences, Seoul National University, San 56-1, Silim-dong, Gwanak-gu, Seoul 151-747, Korea, ${ }^{2}$ Howard Hughes Medical Institute, Columbia University College of Physicians and Surgeons, New York, NY 10032, USA and ${ }^{3}$ Center for Neurobiology and Behavior, Columbia University College of Physicians and Surgeons, New York, NY 10032, USA

Email: Yong-Seok Lee - rhieys@snu.ac.kr; Craig H Bailey - chb1@columbia.edu; Eric R Kandel - erk5@columbia.edu; BongKiun Kaang* - kaang@snu.ac.kr

* Corresponding author
\end{abstract}

Published: 17 June 2008

Molecular Brain 2008, 1:3 doi:10.1 186/1756-6606-1-3
Received: 19 March 2008

Accepted: 17 June 2008

This article is available from: http://www.molecularbrain.com/content/l/I/3

(C) 2008 Lee et al; licensee BioMed Central Ltd.

This is an Open Access article distributed under the terms of the Creative Commons Attribution License (http://creativecommons.org/licenses/by/2.0), which permits unrestricted use, distribution, and reproduction in any medium, provided the original work is properly cited.

\begin{abstract}
Whereas the induction of short-term memory involves only covalent modifications of constitutively expressed preexisting proteins, the formation of long-term memory requires gene expression, new RNA, and new protein synthesis. On the cellular level, transcriptional regulation is thought to be the starting point for a series of molecular steps necessary for both the initiation and maintenance of long-term synaptic facilitation (LTF). The core molecular features of transcriptional regulation involved in the long-term process are evolutionally conserved in Aplysia, Drosophila, and mouse, and indicate that gene regulation by the cyclic AMP response element binding protein (CREB) acting in conjunction with different combinations of transcriptional factors is critical for the expression of many forms of long-term memory. In the marine snail Aplysia, the molecular mechanisms that underlie the storage of long-term memory have been extensively studied in the monosynaptic connections between identified sensory neuron and motor neurons of the gill-withdrawal reflex. One tail shock or one pulse of serotonin (5-HT), a modulatory transmitter released by tail shocks, produces a transient facilitation mediated by the CAMPdependent protein kinase leading to covalent modifications in the sensory neurons that results in an enhancement of transmitter release and a strengthening of synaptic connections lasting minutes. By contrast, repeated pulses of 5-hydroxytryptamine $(5-\mathrm{HT})$ induce a transcription- and translation-dependent long-term facilitation (LTF) lasting more than $24 \mathrm{~h}$ and trigger the activation of a family of transcription factors in the presynaptic sensory neurons including ApCREBI, ApCREB2 and ApC/EBP. In addition, we have recently identified novel transcription factors that modulate the expression of ApC/EBP and also are critically involved in LTF. In this review, we examine the roles of these transcription factors during consolidation of LTF induced by different stimulation paradigms.
\end{abstract}

\section{Introduction}

Memory can be divided into declarative and non-declarative processes. Declarative or explicit memory is the con- scious recall of knowledge about facts and events and is particularly well developed in the vertebrate brain. Nondeclarative or implicit memory is the non-conscious recall 
of skilled behavior and other tasks and includes simple associative forms such as classical conditioning and nonassociative forms such as sensitization [1,2]. During sensitization an animal learns about the properties of a single noxious stimulus enabling the formation of a learned fear response. The cellular and molecular mechanisms that underlie the storage of implicit memory have been most extensively analyzed in the gill- and siphon-withdrawal reflex of marine mollusk Aplysia. This organism offers several unique advantages for the study of learning and memory, such as a relatively simple and tractable central nervous system, large identified neurons, and well-characterized neural circuits related to specific behaviors that can be modified by learning [3]. In Aplysia, sensitization of the gill- and siphon-withdrawal reflex is induced by a strong stimulus to its tail [4]. Repetitive stimuli produce longterm sensitization that lasts days to several weeks whereas a single stimulus induces short-term sensitization lasting only minutes to a few hours $[5,6]$. These two forms of memory can be reconstituted in dissociated sensorymotor neuron cultures by the modulatory neurotransmitter serotonin (5-HT) [7]. A single pulse of 5-HT induces short-term facilitation (STF), whereas five applications of 5-HT induce long-term facilitation (LTF) [8].

In contrast to the short-term synaptic changes, which involve covalent modifications of preexisting molecules leading to an alteration of preexisting synaptic connections, long-term synaptic changes require the synthesis of new macromolecules including mRNAs and proteins [811]. In addition, long-term changes are accompanied by structural modifications including the growth of new synaptic connections between the sensory neurons and their target motor neurons $[12,13]$. These features of long-term, learning-related synaptic plasticity, are highly conserved throughout evolution of the nervous system. A variety of experimental systems, ranging in complexity from Aplysia to rodents, have been used to examine the molecular mechanism underlying long-term synaptic changes $[1,2,10,11]$. In this review, we focus on the role of nuclear transcription factors in the presynaptic sensory neurons of Aplysia during LTF.

\section{A model system for examining the molecular biology of long-term memory - the Aplysia sensory to motor neuron synapse}

In sensory-motor neuron cultures, STF and LTF can be induced by applying 5-HT. One pulse of 5-HT activates PKA and PKC, probably via the activation of different types of $G$ proteins. Activated PKA phosphorylates a potassium channel (S channel), resulting in the elevated influx of calcium leading to an increase in membrane excitability and spike broadening. PKC facilitates the mobilization of synaptic vesicles to the presynaptic active zone. Together, these kinases enhance transmitter release by modifying preexisting molecules [14-17].

LTF induced by repeated pulses of 5-HT requires the synthesis of both new proteins and RNAs. Inhibitors of protein or RNA synthesis selectively block LTF, but not STF when applied within a critical time window that encompasses the training protocol [8]. Analogous to STF, CAMPdependent protein phosphorylation is also involved in LTF and CAMP analogs induce LTF [18]. Bacskai and colleagues first demonstrated that in response to repeated pulses of 5-HT the catalytic subunit of PKA translocates into the nucleus of the presynaptic sensory neuron to activate CREB-1 [19]. Repeated pulses of 5-HT also induce phosphorylation of MAPK and the activated MAPK also translocates into the nucleus of the sensory neuron where it removes the repressive influence of ApCREB-2 [20].

Dash et al. provided the first evidence of the involvement of cAMP-inducible genes expressed during LTF in Aplysia. Microinjection of CRE (cAMP-responsive element) oligonucleotides into the nucleus of sensory neurons selectively blocked 5-HT-induced LTF without affecting shortterm changes [21]. These data first suggested that LTF requires the activation of cAMP-inducible genes, and that CRE oligonucleotides prevent interactions between CREbinding protein (CREB)-related transcription factors and these genes. Using a newly developed gene transfer technique, Kaang and colleagues next showed directly that four or more pulses of 5-HT stimulate CRE-mediated gene expression. Moreover, transcription induced by 5-HT requires the phosphorylation of CREB on Ser ${ }^{119}$ by PKA [22]. Indeed Bartsch et al. went on to show that injection of phosphorylated CREB into the sensory neuron can by itself initiate the long-term process [23]. Collectively these results suggested that a signaling axis composed of CAMPPKA-CREB participates in the molecular cascade leading to the expression of LTF. Subsequent studies confirmed the involvement of a number of related downstream molecules in the induction of LTF, including CAMAP, ApC/ EBP, and ApAF.

\section{ApCREBs - Central modulators of LTF}

In Aplysia, ApCREB1a and ApCREB2 have been characterized as an activator and repressor of LTF, respectively. ApCREB2 is a homolog of mammalian CREB2/ATF4 which is also identified as a transcriptional repressor $[24,25]$. Inhibition of ApCREB2 by injecting antiserum or double-strand RNA (dsRNA) into sensory neurons allowed a single pulse of 5-HT to produce translationdependent LTF and the growth of new synapses $[25,26]$. Conversely, injection of an anti-CREB1 antibody into sensory neurons selectively blocked LTF. Moreover, introduction of the phosphorylated transcriptional activator, ApCREB1a, was sufficient to induce LTF. [23]. PKA-medi- 
ated activation of ApCREB1a stimulated the downstream transcription factor, ApC/EBP, via recruiting CBP and subsequently facilitating histone acetylation [27]. For activation of downstream gene expression by ApCREB1a, the inhibitory constraint of ApCREB2 must be relieved. This de-repression is mediated by the phosphorylation of ApCREB2 by nuclear translocated MAPK [20,28]. Together these results suggest that LTF requires not only the activation of memory-enhancer genes but also the inactivation of memory-suppressor genes. Moreover, CREBs appear to play a critical role in maintaining the dynamic balance between these positive and negative factors.

CREB, a basic leucine zipper transcription factor, is also reported to be involved in long-term plasticity in the nervous systems of other organisms, including fly and mouse. In transgenic Drosophila, expression of dCREB2b led to the blockage of long-term memory, whereas dCREB2a facilitated memory $[29,30]$. Similarly, CREB-deficient mice displayed impaired long-term potentiation (LTP) and longterm memory [31]. The threshold for late phase LTP was lowered in the hippocampus from mice expressing the constitutively active form of CREB [32].

\section{ApC/EBP - A key downstream gene for LTF}

In view of the critical roles of CREB in memory consolidation, characterization of its downstream effectors has been a major focus of research in both invertebrate and vertebrate learning models [33-35]. In Aplysia the CCAAT enhancer-binding protein (ApC/EBP), an immediate early gene during the consolidation phase of LTF, was found by Alberini et al. to be a downstream target of ApCREB1 [36]. The C/EBP family of transcription factors contains a basic leucine-zipper domain. Specifically, expression of ApC/ EBP is rapidly induced in response to 5-HT treatment and this occurs to an immediate early gene in a translationindependent manner [36]. Inhibition of ApC/EBP by injection of ERE oligonucleotides, ApC/EBP antiserum or dsRNA into the sensory neurons in sensory-to-motor neuron cultures during a critical time window blocked 5-HTinduced LTF [36,37]. Moreover, a single pulse of 5-HT which normally induces only STF, produces LTF when ApC/EBP is overexpressed in the sensory neuron [37]. These findings support the idea that ApC/EBP is both necessary and sufficient to consolidate short-term memory into long-term memory. However, since overexpression of ApC/EBP alone does not induce LTF, an additional component must be required for converting STF to LTF. Lee and colleagues used the RNA interference technique designed to block the function of ApC/EBP and similarly found that this blocked LTF in Aplysia [26,37]. Moreover, in addition to these studies in Aplysia, C/EBP $\beta$ and $-\delta$ are induced in the rodent hippocampus after inhibitory avoidance learning, suggesting that C/EBPs are highly conserved molecular components of the CREB-dependent signal pathway involved in memory consolidation [38].

ApC/EBP is also known to be induced by the neural activity. The depolarization-induced ApC/EBP induction appears to be mediated by transient induction of the nucleolar protein, ApLLP, which was recently characterized as a novel transcription factor induced by neural activity in Aplysia sensory neuron [39]. Kim et al. also showed that a single pulse of 5-HT can produce LTF when the synapse is pretreated with high potassium solution. LTF induced by this protocol was completely blocked by the injection of anti-ApLLP or anti-ApC/EBP antibody [39].

One important feature of immediate early genes such as $\mathrm{ApC} / \mathrm{EBP}$, is that their expression is tightly regulated within a specific and narrow time window. ApC/EBP mRNA displays a peak expression at $2 \mathrm{~h}$ after induction that rapidly returns to the basal levels [36]. Recent studies have found that the ApC/EBP 3' UTR contains putative AU-rich element (ARE) sequences which are cis-acting regulatory elements commonly found in labile mRNAs [40]. Different sets of ARE binding proteins may interact with ApC/EBP mRNA to regulate its stability and/or translatability [41-43]. Yim et al. found that an ARE binding protein, ApELAV binds to and stabilizes ApC/EBP mRNA, suggesting that post-transcriptional regulation of $\mathrm{ApC} /$ EBP may also play an important role during LTF [40].

\section{ApAF - A binding partner for both ApCREB2 and ApC/EBP}

Both ApC/EBP and ApCREBs are transcription factors containing the basic leucine zipper (bZIP) domain in the Cterminus. This domain is involved in both DNA binding and multimerization $[23,25,44]$. Using the bZIP domain of ApC/EBP as bait, Bartsch and colleagues screened a cDNA library to identify additional transcription factors acting downstream of ApCREB1. In this fashion, they cloned a novel transcription factor ApAF, whose activity is regulated by PKA [45]. In contrast to ApC/EBP, which is induced in response to 5-HT, ApAF is a constitutively expressed gene. Interestingly, an in vitro binding assay revealed that ApAF interacts with both ApCREB2 and ApC/EBP, but not ApCREB1. Inhibition of ApAF by injection of a specific antibody blocked LTF induced by repeated pulses of 5-HT, suggesting that ApAF is necessary for LTF. Previously, it had been found that injection of phosphorylated ApCREB1a by itself or anti-ApCREB2 antibody combined with a single pulse of 5-HT induced LTF that phenocopied $5 \times 5$-HT-induced LTF $[23,25]$. ApAF is involved in both forms of LTF: an anti-ApAF antibody blocked LTF induced by phosphorylated CREB1a as well as that by ApCREB2 antibody injection paired with one pulse of 5-HT. Moreover, overexpression of ApAF 
enhanced both LTF induced by $5 \times 5$-HT and ApCREB2 antibody with $1 \times 5$-HT [45]. Thus, ApAF may be a potential memory enhancer gene downstream of ApCREB1 and ApCREB2.

To determine whether ApC/EBP is the critical partner of ApAF, Lee and colleagues investigated the effects of silencing the ApAF gene on LTF induced by ApC/EBP overexpression paired with one pulse of 5-HT [46]. ApAF inhibition by dsRNA completely blocked LTF induced by both ApC/EBP overexpression and $5 \times 5$-HT. In combination with a single pulse of 5-HT, the ApAF-ApC/EBP heterodimer produced LTF, even in the absence of CRE- and CREB-mediated gene expression. These results provide direct evidence that the ApAF-ApC/EBP heterodimer is a key downstream effecter of ApCREB. Furthermore, ApAF enhances ERE-mediated gene expression by cooperating with ApC/EBP and phosphorylation at Ser-266 of ApAF by PKA is required for activation of the ApAF-ApC/EBP heterodimer during 5-HT-induced LTF [46]. These data explain why ApC/EBP overexpression in the absence of 5HT could not convert STF to LTF. The single pulse of 5-HT in ApC/EBP-induced LTF possibly functions in triggering the phosphorylation of ApAF by activated PKA.

Lee et al. also examined the role of Ser-266 phosphorylation of ApAF in the relief of ApCREB2 repression [46]. Overexpression of a dominant negative mutant of ApAF which cannot be phosphorylated at Ser-266 completely blocked both LTF induced by $5 \times 5$-HT and that by the ApC/EBP overexpression combined with $1 \times 5$-HT. Moreover, this mutant restored ApCREB1-mediated gene expression and 5-HT-induced LTF repressed by ApCREB2 as efficiently as its wild type counterpart, suggesting that Ser-266 phosphorylation of ApAF is not required to relieve ApCREB2 repression [46]. However, the precise signaling pathway that regulates the interactions between ApAF and ApCREB2 remains to be characterized. Taken together, these studies of ApAF indicate that transcriptional regulation of memory consolidation is quite diverse and can recruit both direct and indirect interactions between transcription factors.

\section{CAMAP - A retrograde signal from the membrane to the CRE promoter}

Neurons display a distinct highly differentiated form which consists of three basic compartments: a cell body or soma which contains the nucleus harboring genomic information and two types of processes axons and dendrites. Dendrites are input elements of the neuron. Together with the cell body they receive synaptic contacts from other neurons. Axons are the output elements of the neuron. The branches of each axon form numerous synaptic connections with other neurons. It is well known that long-term synaptic plasticity requires the synthesis of both new RNAs and proteins. This raises three fundamental questions: 1) Does synaptic plasticity always occur in a cell-wide manner? 2) If not, how does the nucleus identify the correct synapses for delivery of gene products to achieve synapse-specific plasticity? 3) Is the nucleus the only site responsible for transcription and translation?

To address the question of synapse specificity, Martin and her colleagues developed a new culture system in Aplysia consisting of a bifurcated Aplysia sensory neuron which makes synapses with two spatially separated motor neurons in culture [47]. They found that 5-HT can induce branch-specific LTF that was dependent on CREB activation in the nucleus of presynaptic sensory neuron. Branchspecific LTF was also accompanied by synapse-specific growth of new sensory neuron synapses. These studies highlight the importance of a retrograde signal propagating from the stimulated synaptic site to the nucleus. The CREB-downstream molecules produced in the cell body can be captured by other synapses which have been tagged or marked. This synaptic mark is PKA-dependent; however, rapamycin-dependent local protein synthesis is required for LTF to persist for more than $72 \mathrm{~h}[47,48]$. Further molecular studies have dissected the characteristics of the retrograde signal and the synaptic mark.

An early step in the growth of new synaptic connections is the internalization of an NCAM immunoglobulin-related cell adhesion molecule - apCAM. In recent studies Lee et al. addressed the question: How does this internalization of apCAM relate to the activation of transcription? Lee found that CAMAP is an interacting partner of apCAM in the sensory neuron. CAMAP serves as a transcriptional coactivator that is also a crucial retrograde signaling molecule involved in the initiation phase of LTF [49]. ApCAM is down-regulated upon application of 5-HT [50]. Serotonin leads to clathrin-mediated endocytosis of the transmembrane isoform of apCAM (TM-ApCAM) from the surface membrane of sensory neurons and this internalization depends on phosphorylation of its cytoplasmic tail by MAPK [51,52]. When TM-apCAM was overexpressed in sensory neurons, five pulses of 5-HT failed to produce synaptic facilitation or enhancement of synaptic growth, suggesting that down-regulation of apCAM is required for both LTF and the presynaptic structural changes induced by 5 -HT [53]. Whereas MAPK is known to be involved in apCAM downregulation, the subcellular mechanisms responsible for apCAM internalization remain to be characterized. CAMAP (apCAM-Associated Protein) was cloned by yeast two-hybrid screening using the cytoplasmic tail of TM-ApCAM as bait [49]. CAMAP is colocalized with apCAM at the surface of the plasma membrane in the basal state and is translocated to the nucleus of sensory neuron after treatment with 5-HT. Nuclear translocation and dissociation from apCAM are modulated by PKA- 
mediated phosphorylation of CAMAP. A phosphorylation-mimicking mutant of CAMAP can dissociate from apCAM and translocate to the nucleus in the absence of 5HT stimulation [49]. How does CAMAP translocate to the nucleus? Importins, which transport cargo molecules from the cytosol to nucleus, are critically involved in synaptic plasticity in both Aplysia and rodent brain [54]. This raises the possibility that importin may transport CAMAP into the nucleus of the sensory neuron.

In view of nuclear translocation of CAMAP from the plasma membrane, Lee et al. suggested that CAMAP acts as a retrograde signaling molecule in the induction of LTF. To act as a retrograde signal, a molecule must meet several criteria, specifically, 1) it should translocate into the nucleus from the synaptic site in response to stimuli that induce synaptic plasticity, such as LTF and LTP, 2) it should play a specific function in the nucleus, and 3 ) blockage of its translocation and function should inhibit the long-term synaptic change. CAMAP appears to fulfill all of the above requirements. First, CAMAP translocates to the nucleus following both cell-wide and synapse-specific applications of 5-HT. After 5-HT treatment, the mobility of CAMAP is significantly increased at the distal neurites of sensory neurons. Second, CAMAP binds to APCREB 1 in the nucleus, where it acts as a transcriptional co-activator that can induce ApC/EBP expression. Interestingly, the N-terminal region, but not full-length CAMAP displayed transcriptional activity. The C-terminus of CAMAP appears to act as an autoinhibitory domain. This inhibitory clamp is relieved by PKA phosphorylation of CAMAP. Details of the molecular mechanisms that underlie the co-activator function of CAMAP and the PKA phosphorylation-dependent restoration of transcriptional activity of the N-terminus of CAMAP are currently not known. Finally, blockage of CAMAP expression by dsRNA completely suppresses 5-HT-induced ApC/EBP upregulation and LTF. Moreover, CAMAP dsRNA blocks synapsespecific LTF induced by local application of 5-HT at the synapse. Taken together, these results indicate that CAMAP is a critical retrograde messenger in the initiation of LTF.

The precise role of CAMAP in the internalization of apCAM remains unclear. We have recently found that overexpression of mutant CAMAP that cannot be phosphorylated by PKA impaired the 5-HT-induced internalization of apCAM [49]. This finding suggests that CAMAP tethers apCAM to the plasma membrane in the basal state, and that the phosphorylation and nuclear translocation of CAMAP are necessary for the subsequent down-regulation of apCAM.

\section{Chromatin alteration and epigenetic changes in gene expression with memory storage}

Although epigenetic mechanisms were widely known to be involved in the formation and long-term storage of cellular information in response to transient environmental signals, the discovery of their putative relavance in adult brain function is relatively recent $[27,55]$. The epigenetic marking of chromatin, such as histone modification, chromatin remodeling and the activity of retrotransposons, may have long-term consequences in the transcriptional regulation of specific loci involved for longterm synaptic changes [56].

The contribution of histone tail acetylation, a modification that favors transcription and is associated with active loci, to LTF formation was first revealed by the study of Guan et al. in Aplysia neurons [27]. This study found that both facilitatory and inhibitory stimuli bidirectionally alter the acetylation stage and structure of promoters driven by the expression of genes involved in the maintenance of LTF, such as ApC/EBP. It also demonstrated that enhancing histone acetylation with histone deacetylase (HDAC) inhibitors facilitates the induction of LTF (Guan et al. 2002). HDAC inhibitors have recently been shown to have similar effects during L-LTP in the Schaffer collateral pathway of mammals and to enhance memory formation in hippocampus-dependent tasks [57-61]. HDAC inhibitor, sodium butyrate, has been shown to induce the growth of new dendrites and synapses, which might be an underlying mechanism of its memory enhancing effects [61]. Moreover, mice with reduced histone acetyltransferase activity, such as different mouse models for Rubinstein-Taybi mental retardation syndrome, have deficits in both long-lasting forms of memory and LTP $[57,58,62,63]$. These results indicate that critical chromatin changes occur during the formation of long-term memory and that these changes are required for the stable maintenance of these memories.

\section{Perspectives}

During the last decade molecular studies have increased our understanding of the signaling and regulatory mechanisms that underlie LTF (Figure 1). Several research groups have generated a variety of cellular models of longterm memory by investigating 5-HT-induced LTF and other forms of long-term synaptic changes induced by different stimulation paradigms in Aplysia.

Recently, operant conditioning was demonstrated in the gill-withdrawal and feeding behavior in Aplysia and an electrophysiological study revealed that operant and classical conditioning of feeding behavior differentially modify the intrinsic excitability of an identified neuron [6466]. PolyADP-ribose-polymerase 1 (PARP1) facilitates the transcription of long-term memory related genes by 


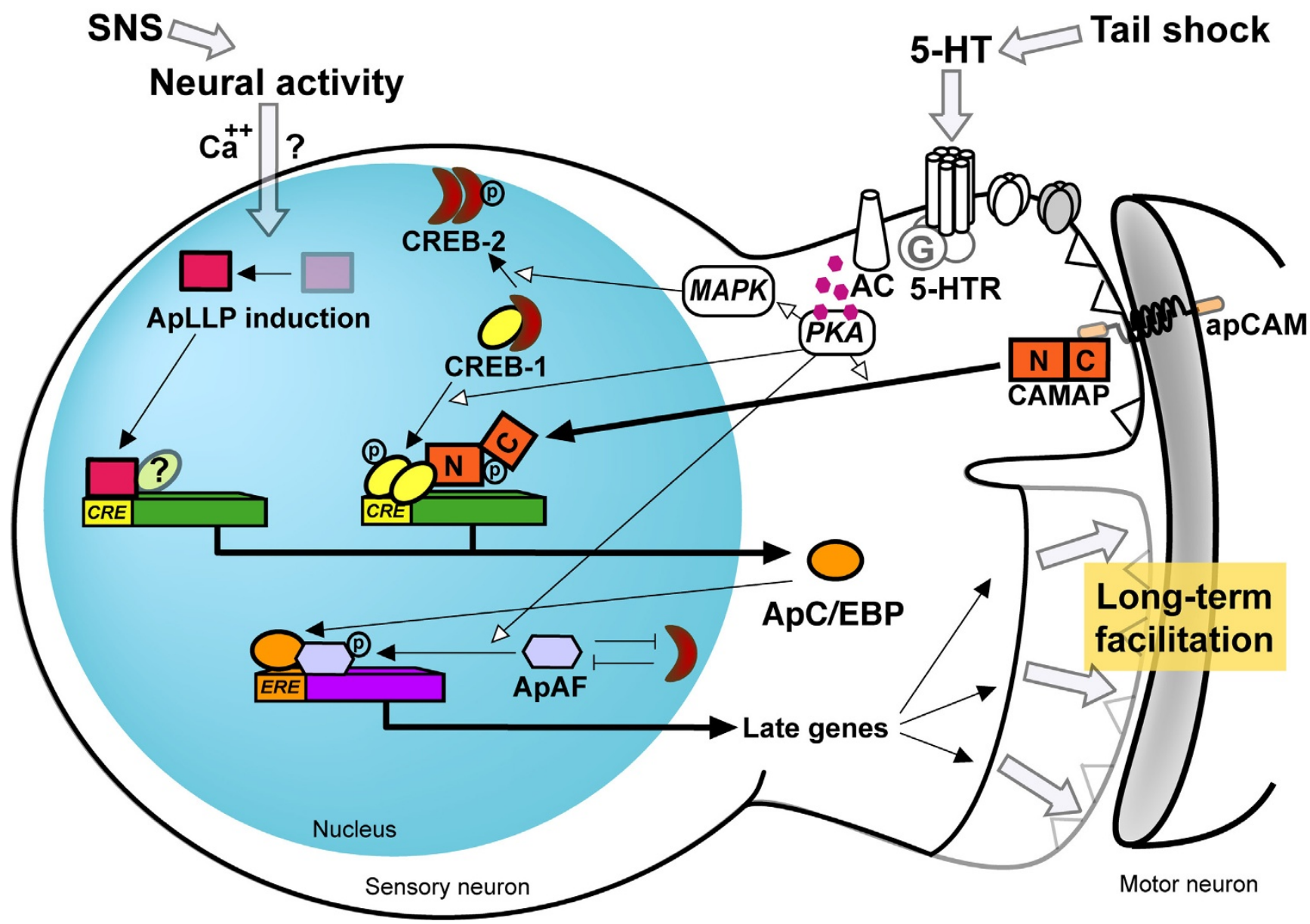

Figure I

Schematic model of signaling pathways underlying long-term facilitation in Aplysia sensory neuron. The repeated treatments with neurotransmitter 5-HT activate a G-protein coupled receptor that stimulates adenylyl cyclase, which in turn activates PKA. MAPK are also activated and translocates into the nucleus. At the synaptic site, PKA stimulates the nuclear translocation of the retrograde signal molecule CAMAP via phosphorylating its Ser ${ }^{148}$. This phosphorylation results in both the dissociation from TM-apCAM and the restoration of its transcriptional activity from autoinhibition. In the nucleus, MAPK phosphorylates CREB2 which represses CREBI and APAF in the absence of 5-HT. Once freed from CREB2 and stimulated by PKA, CREBI forms a homodimer to activate the downstream target gene, ApC/EBP. Translocated CAMAP acts as a co-activator of CREBI. ApC/EBP interacts with ApAF that is activated by PKA to form a core downstream effector of CREBI. ApC/EBP-ApAF heterodimer induces the late genes which are critical for the consolidation and maintenance of LTF. Robust neural activity induces and activates the transcription factor, ApLLP in the nucleus in a calcium-dependent manner. ApLLP induces ApC/EBP expression and lowers the threshold for LTF induction. Elucidating the downstream molecule of ApC/EBP remains to be challenged. SNS, strong noxious stimulus.

decondensing chromatin structure in neurons that mediate operant conditioning [67]. However, the transcription factors involved in this behavioral modification are yet to be identified.

Interestingly, ApC/EBP is the common downstream molecule of the novel transcription factors, ApLLP and CAMAP. ApC/EBP, which pairs with ApAF, could activate the transcription of effector genes critically involved in the consolidation and maintenance of long-term memory [36]. Genes encoding structural proteins, such as clathrin light chain and the chaperon $\mathrm{BiP}$ were identified as late effector genes $[68,69]$. The elongation factor 1 alpha was also suggested to be essential for maintaining newly formed synapses [70]. However, the number of late genes identified thus far represents only a beginning. Since the DNA-binding motifs of ApC/EBP and ApAF homodimer or heterodimer have been analyzed, completion of the ongoing Aplysia genome sequencing project should facilitate the identification of other novel late effector molecules [45]. 
Recently, advances have also been made in clarifying the molecular mechanisms that contribute to learning-related synaptic plasticity in the mammalian brain, particularly those that underlie the induction and expression of LTP and LTD $[71,72]$. When it comes to transcriptional regulation, however, our current understanding is far from complete. Aplysia was the first organism in which cAMP was shown to play a critical role in learning-related synaptic plasticity $[28,73]$. Since signaling cascades that underlie the expression of long-term memory are surprisingly well conserved throughout the species, insights from the molecular studies of Aplysia should provide an important foundation for future studies into the transcriptional regulation of memory formation in the more complex mammalian brain.

\section{Authors' contributions}

All authors participated in developing the ideas, the writing, discussion and integration of the information. All authors read and approved the final manuscript.

\section{Acknowledgements}

Our special thanks go to Hyoung Kim for the graphic work of the figure. This work was supported by the Creative Research Initiative Program of the Korean Ministry of Science and Technology (to B.-K.K.).

\section{References}

I. Milner B, Squire LR, Kandel ER: Cognitive neuroscience and the study of memory. Neuron 1998, 20(3):445-468.

2. Bailey CH, Bartsch D, Kandel ER: Toward a molecular definition of long-term memory storage. Proc Natl Acad Sci U S A 1996, 93(24): 1 3445-13452.

3. Kandel ER: Behavioral biology of Aplysia : a contribution to the comparative study of opisthobranch molluscs /Eric R. Kandel. San Francisco, W. H. Freeman; 1979:xiii, 463 p..

4. Kandel ER: Cellular basis of behavior. In A series of books in psychology New York, W.H. Freeman and company; 1976.

5. Castellucci VF, Frost WN, Goelet P, Montarolo PG, Schacher S, Morgan JA, Blumenfeld $\mathrm{H}$, Kandel ER: Cell and molecular analysis of long-term sensitization in Aplysia. J Physiol (Paris) 1986, 8I(4):349-357.

6. Pinsker HM, Hening WA, Carew TJ, Kandel ER: Long-term sensitization of a defensive withdrawal reflex in Aplysia. Science 1973, 182(1 16): 1039-1042.

7. Rayport SG, Schacher S: Synaptic plasticity in vitro: cell culture of identified Aplysia neurons mediating short-term habituation and sensitization. J Neurosci 1986, 6(3):759-763.

8. Montarolo PG, Goelet P, Castellucci VF, Morgan J, Kandel ER, Schacher S: A critical period for macromolecular synthesis in long-term heterosynaptic facilitation in Aplysia. Science 1986, 234(478I): $1249-1254$.

9. Castellucci VF, Blumenfeld H, Goelet P, Kandel ER: Inhibitor of protein synthesis blocks long-term behavioral sensitization in the isolated gill-withdrawal reflex of Aplysia. J Neurobiol 1989, 20(I): I-9.

10. Kandel ER: Genes, nerve cells, and the remembrance of things past. J Neuropsychiatry Clin Neurosci 1989, I (2): 103-125.

II. Kandel ER: The molecular biology of memory storage: a dialogue between genes and synapses. Science 200I, 294(5544): $1030-1038$.

12. Bailey CH, Montarolo P, Chen M, Kandel ER, Schacher S: Inhibitors of protein and RNA synthesis block structural changes that accompany long-term heterosynaptic plasticity in Aplysia. Neuron 1992, 9(4):749-758.

13. Glanzman DL, Kandel ER, Schacher S: Target-dependent structural changes accompanying long-term synaptic facilitation in Aplysia neurons. Science 1990, 249(4970):799-802.
14. Byrne JH, Kandel ER: Presynaptic facilitation revisited: state and time dependence. J Neurosci 1996, 16(2):425-435.

15. Klein M, Camardo J, Kandel ER: Serotonin modulates a specific potassium current in the sensory neurons that show presynaptic facilitation in Aplysia. Proc Natl Acad Sci U S A 1982, 79(18):57|3-57|7.

16. Sugita S, Baxter DA, Byrne JH: Activators of protein kinase $\mathbf{C}$ mimic serotonin-induced modulation of a voltage-dependent potassium current in pleural sensory neurons of Aplysia. J Neurophysiol 1994, 72(3): 1240-1249.

17. Baxter DA, Byrne JH: Differential effects of cAMP and serotonin on membrane current, action-potential duration, and excitability in somata of pleural sensory neurons of Aplysia. J Neurophysiol 1990, 64(3):978-990.

18. Schacher S, Castellucci VF, Kandel ER: CAMP evokes long-term facilitation in Aplysia sensory neurons that requires new protein synthesis. Science 1988, 240(4859): 1667-1669.

19. Bacskai BJ, Hochner B, Mahaut-Smith M, Adams SR, Kaang BK, Kandel $E R$, Tsien RY: Spatially resolved dynamics of CAMP and protein kinase A subunits in Aplysia sensory neurons. Science 1993, 260(5 I 05):222-226.

20. Martin KC, Michael D, Rose JC, Barad M, Casadio A, Zhu H, Kandel ER: MAP kinase translocates into the nucleus of the presynaptic cell and is required for long-term facilitation in Aplysia. Neuron 1997, 18(6):899-912.

21. Dash PK, Hochner B, Kandel ER: Injection of the CAMP-responsive element into the nucleus of Aplysia sensory neurons blocks long-term facilitation. Nature 1990, 345(6277):718-72 I.

22. Kaang BK, Kandel ER, Grant SG: Activation of cAMP-responsive genes by stimuli that produce long-term facilitation in Aplysia sensory neurons. Neuron 1993, 10(3):427-435

23. Bartsch D, Casadio A, Karl KA, Serodio P, Kandel ER: CREB I encodes a nuclear activator, a repressor, and a cytoplasmic modulator that form a regulatory unit critical for long-term facilitation. Cell 1998, 95(2):2II-223.

24. Karpinski BA, Morle GD, Huggenvik J, Uhler MD, Leiden JM: Molecular cloning of human CREB-2: an ATF/CREB transcription factor that can negatively regulate transcription from the cAMP response element. Proc Natl Acad Sci U S A 1992, 89(I I):4820-4824.

25. Bartsch D, Ghirardi M, Skehel PA, Karl KA, Herder SP, Chen M, Bailey $\mathrm{CH}$, Kandel ER: Aplysia CREB2 represses long-term facilitation: relief of repression converts transient facilitation into long-term functional and structural change. Cell 1995, 83(6):979-992.

26. Lee JA, Kim H, Lee YS, Kaang BK: Overexpression and RNA interference of Ap-cyclic AMP-response element binding protein-2, a repressor of long-term facilitation, in Aplysia kurodai sensory-to-motor synapses. Neurosci Lett 2003, 337(I):9-12.

27. Guan Z, Giustetto M, Lomvardas S, Kim JH, Miniaci MC, Schwartz JH, Thanos D, Kandel ER: Integration of long-term-memoryrelated synaptic plasticity involves bidirectional regulation of gene expression and chromatin structure. Cell 2002, III (4):483-493.

28. Cedar H, Kandel ER, Schwartz JH: Cyclic adenosine monophosphate in the nervous system of Aplysia californica. I. Increased synthesis in response to synaptic stimulation. J Gen Physiol 1972, 60(5):558-569.

29. Yin JC, Del Vecchio M, Zhou H, Tully T: CREB as a memory modulator: induced expression of a dCREB2 activator isoform enhances long-term memory in Drosophila. Cell 1995, 8I(I): $107-115$.

30. Yin JC, Wallach JS, Del Vecchio M, Wilder EL, Zhou H, Quinn WG, Tully T: Induction of a dominant negative CREB transgene specifically blocks long-term memory in Drosophila. Cell 1994, 79(I):49-58.

31. Bourtchuladze R, Frenguelli B, Blendy J, Cioffi D, Schutz G, Silva AJ: Deficient long-term memory in mice with a targeted mutation of the cAMP-responsive element-binding protein. Cell 1994, 79(1):59-68.

32. Barco A, Alarcon JM, Kandel ER: Expression of constitutively active CREB protein facilitates the late phase of long-term potentiation by enhancing synaptic capture. Cell 2002, 108(5):689-703. 
33. Barco A, Patterson S, Alarcon JM, Gromova P, Mata-Roig M, Morozov A, Kandel ER: Gene expression profiling of facilitated L-LTP in VP I6-CREB mice reveals that BDNF is critical for the maintenance of LTP and its synaptic capture. Neuron 2005, 48(I): $123-137$.

34. Alberini CM, Ghirardi M, Huang YY, Nguyen PV, Kandel ER: A molecular switch for the consolidation of long-term memory: cAMP-inducible gene expression. Ann N Y Acad Sci 1995, 758:26I-286.

35. Silva AJ, Kogan JH, Frankland PW, Kida S: CREB and memory. Annu Rev Neurosci 1998, 21:127-148.

36. Alberini CM, Ghirardi M, Metz R, Kandel ER: C/EBP is an immediate-early gene required for the consolidation of long-term facilitation in Aplysia. Cell 1994, 76(6): 1099-III4.

37. Lee JA, Kim HK, Kim KH, Han JH, Lee YS, Lim CS, Chang DJ, Kubo $T$, Kaang BK: Overexpression of and RNA interference with the CCAAT enhancer-binding protein on long-term facilitation of Aplysia sensory to motor synapses. Learn Mem 200I, 8(4):220-226.

38. Taubenfeld SM, Wiig KA, Monti B, Dolan B, Pollonini G, Alberini CM: Fornix-dependent induction of hippocampal CCAAT enhancer-binding protein [beta] and [delta] Co-localizes with phosphorylated CAMP response element-binding protein and accompanies long-term memory consolidation. J Neurosci 200I, 2I(I):84-9I.

39. Kim H, Lee SH, Han JH, Lee JA, Cheang YH, Chang DJ, Lee YS, Kaang BK: A nucleolar protein ApLLP induces ApC/EBP expression required for long-term synaptic facilitation in aplysia neurons. Neuron 2006, 49(5):707-7|8.

40. Yim SJ, Lee YS, Lee JA, Chang DJ, Han JH, Kim H, Park H, Jun H, Kim VN, Kaang BK: Regulation of ApC/EBP mRNA by the Aplysia AU-rich element-binding protein, ApELAV, and its effects on 5-hydroxytryptamine-induced long-term facilitation. J Neurochem 2006, 98(2):420-429.

41. Xu N, Chen CY, Shyu AB: Versatile role for hnRNP D isoforms in the differential regulation of cytoplasmic mRNA turnover. Mol Cell Biol 200I, 2 I(20):6960-697I.

42. Bevilacqua A, Ceriani MC, Capaccioli S, Nicolin A: Post-transcriptional regulation of gene expression by degradation of messenger RNAs. / Cell Physiol 2003, 195(3):356-372.

43. Guhaniyogi J, Brewer $\mathrm{G}$ : Regulation of mRNA stability in mammalian cells. Gene 200I, 265(I-2): II-23.

44. Mayr B, Montminy M: Transcriptional regulation by the phosphorylation-dependent factor CREB. Nat Rev Mol Cell Biol 200I, 2(8):599-609.

45. Bartsch D, Ghirardi M, Casadio A, Giustetto M, Karl KA, Zhu H, Kandel ER: Enhancement of memory-related long-term facilitation by ApAF, a novel transcription factor that acts downstream from both CREBI and CREB2. Cell 2000, 103(4):595-608.

46. Lee JA, Lee SH, Lee C, Chang DJ, Lee Y, Kim H, Cheang YH, Ko HG, Lee YS, Jun H, Bartsch D, Kandel ER, Kaang BK: PKA-activated ApAF-ApC/EBP heterodimer is a key downstream effector of APCREB and is necessary and sufficient for the consolidation of long-term facilitation. J Cell Biol 2006, 174(6):827-838.

47. Martin KC, Casadio A, Zhu H, Yaping E, Rose JC, Chen M, Bailey CH, Kandel ER: Synapse-specific, long-term facilitation of aplysia sensory to motor synapses: a function for local protein synthesis in memory storage. Cell 1997, 91 (7):927-938.

48. Casadio A, Martin KC, Giustetto M, Zhu H, Chen M, Bartsch D, Bailey $\mathrm{CH}$, Kandel ER: A transient, neuron-wide form of CREB-mediated long-term facilitation can be stabilized at specific synapses by local protein synthesis. Cell 1999, 99(2):22।-237.

49. Lee SH, Lim CS, Park H, Lee JA, Han JH, Kim H, Cheang YH, Lee YS, Ko HG, Jang DH, Miniaci MC, Bartsch D, Kim E, Bailey CH, Kandel ER, Kaang BK: Nuclear Translocation of CAM-Associated Protein Activates Transcription for Long-Term Facilitation in Aplysia. Cell 2007, I 29(4):80I-8I2.

50. Mayford M, Barzilai A, Keller F, Schacher S, Kandel ER: Modulation of an NCAM-related adhesion molecule with long-term synaptic plasticity in Aplysia. Science 1992, 256(5057):638-644.

5I. Bailey CH, Chen M, Keller F, Kandel ER: Serotonin-mediated endocytosis of apCAM: an early step of learning-related synaptic growth in Aplysia. Science 1992, 256(5057):645-649.

52. Bailey CH, Kaang BK, Chen M, Martin KC, Lim CS, Casadio A, Kandel ER: Mutation in the phosphorylation sites of MAP kinase blocks learning-related internalization of apCAM in Aplysia sensory neurons. Neuron 1997, 18(6):913-924.

53. Han JH, Lim CS, Lee YS, Kandel ER, Kaang BK: Role of Aplysia cell adhesion molecules during 5-HT-induced long-term functional and structural changes. Learn Mem 2004, II (4):42I-435.

54. Thompson KR, Otis KO, Chen DY, Zhao Y, O'Dell TJ, Martin KC: Synapse to nucleus signaling during long-term synaptic plasticity; a role for the classical active nuclear import pathway. Neuron 2004, 44(6):997-I009.

55. Levenson JM, Sweatt JD: Epigenetic mechanisms in memory formation. Nat Rev Neurosci 2005, 6(2): 108-II8.

56. Hsieh J, Gage FH: Chromatin remodeling in neural development and plasticity. Curr Opin Cell Biol 2005, I 7(6):664-67I.

57. Alarcon JM, Malleret G, Touzani K, Vronskaya S, Ishii S, Kandel ER, Barco A: Chromatin acetylation, memory, and LTP are impaired in CBP+/- mice: a model for the cognitive deficit in Rubinstein-Taybi syndrome and its amelioration. Neuron 2004, 42(6):947-959.

58. Korzus E, Rosenfeld MG, Mayford M: CBP histone acetyltransferase activity is a critical component of memory consolidation. Neuron 2004, 42(6):961-972.

59. Levenson JM, O'Riordan KJ, Brown KD, Trinh MA, Molfese DL, Sweatt JD: Regulation of histone acetylation during memory formation in the hippocampus. J Biol Chem 2004, 279(39):40545-40559.

60. Yeh SH, Lin CH, Gean PW: Acetylation of nuclear factor-kappaB in rat amygdala improves long-term but not short-term retention of fear memory. Mol Pharmacol 2004, 65(5): $1286-1292$.

61. Fischer A, Sananbenesi F, Wang X, Dobbin M, Tsai LH: Recovery of learning and memory is associated with chromatin remodelling. Nature 2007, 447(7 I 4I): 178- 182.

62. Wood MA, Kaplan MP, Park A, Blanchard EJ, Oliveira AM, Lombardi TL, Abel T: Transgenic mice expressing a truncated form of CREB-binding protein (CBP) exhibit deficits in hippocampal synaptic plasticity and memory storage. Learn Mem 2005, I 2(2): I | |- I 9.

63. Bourtchouladze R, Lidge R, Catapano R, Stanley J, Gossweiler S, Romashko D, Scott R, Tully T: A mouse model of RubinsteinTaybi syndrome: defective long-term memory is ameliorated by inhibitors of phosphodiesterase 4. Proc Natl Acad SciU S A 2003, I00(18): 105 | 8-10522.

64. Lorenzetti FD, Mozzachiodi R, Baxter DA, Byrne JH: Classical and operant conditioning differentially modify the intrinsic properties of an identified neuron. Nat Neurosci 2006, 9(I):17-19.

65. Brembs B, Lorenzetti FD, Reyes FD, Baxter DA, Byrne JH: Operant reward learning in Aplysia: neuronal correlates and mechanisms. Science 2002, 296(5573): I 706-1709.

66. Hawkins RD, Clark GA, Kandel ER: Operant conditioning of gill withdrawal in Aplysia. J Neurosci 2006, 26(9):2443-2448.

67. Cohen-Armon M, Visochek L, Katzoff A, Levitan D, Susswein AJ, Klein $\mathrm{R}$, Valbrun M, Schwartz JH: Long-term memory requires polyADP-ribosylation. Science 2004, 304(5678): $1820-1822$.

68. Hu Y, Barzilai A, Chen M, Bailey CH, Kandel ER: 5-HT and cAMP induce the formation of coated pits and vesicles and increase the expression of clathrin light chain in sensory neurons of aplysia. Neuron 1993, 10(5):921-929.

69. Kuhl D, Kennedy TE, Barzilai A, Kandel ER: Long-term sensitization training in Aplysia leads to an increase in the expression of BiP, the major protein chaperon of the ER. J Cell Biol 1992, I I9(5): 1069-1076.

70. Giustetto M, Hegde AN, Si K, Casadio A, Inokuchi K, Pei W, Kandel $E R$, Schwartz JH: Axonal transport of eukaryotic translation elongation factor I alpha mRNA couples transcription in the nucleus to long-term facilitation at the synapse. Proc Natl Acad Sci U S A 2003, 100(23): 13680-13685.

7I. Malenka RC, Bear MF: LTP and LTD: an embarrassment of riches. Neuron 2004, 44(I):5-2I.

72. Rodrigues SM, Schafe GE, LeDoux JE: Molecular mechanisms underlying emotional learning and memory in the lateral amygdala. Neuron 2004, 44(I):75-91.

73. Brunelli M, Castellucci V, Kandel ER: Synaptic facilitation and behavioral sensitization in Aplysia: possible role of serotonin and cyclic AMP. Science 1976, 194(4270): I 178-1 I81. 